\title{
Lagrangian transport in a circular lake: effect of nonlinearity and the second vertical mode
}

\author{
T. Sakai ${ }^{1, *}$ and L. G. Redekopp ${ }^{1}$ \\ ${ }^{1}$ Department of Aerospace \& Mechanical Engineering, University of Southern California, Los Angeles, \\ CA 90089-1191, USA \\ "now at: School of Civil and Environmental Engineering, Cornell University, Ithaca, NY 14853-3501, USA
}

Received: 21 June 2011 - Revised: 14 October 2011 - Accepted: 25 October 2011 - Published: 28 October 2011

\begin{abstract}
Effects of the second vertical mode and nonlinearity of the background flow field on the Lagrangian transport of particle clouds are studied by employing a wind-forced linear hydrostatic model and a weakly-nonlinear, weaklynonhydrostatic evolution model. It is confirmed that Kelvin waves primarily advect particles near the basin perimeter in a cyclonic direction, and Poincaré waves primarily advect particles in off-shore (radial) directions in a manner that is oscillatory in time with frequencies near the inertial period. The internal current associated with the second vertical mode is usually far smaller than that associated with the energetically dominant, lowest vertical mode. However, because of the disparately slow eigenspeed of the vertical mode-two Kelvin wave, the resultant particle transport associated with the vertical mode-two flow near the basin perimeter can drive transport that is comparable with that associated with the Kelvin wave of the lowest vertical mode. It is discovered that nonlinear interaction between the Kelvin-Poincaré wave pair can give birth to a solitary-like wave of large amplitude in an offshore region. This new type of wave generates a large current and co-propagates with the Kelvin wave in a cyclonic direction and, eventually, can cause a burst of particle transport in an off-shore direction.
\end{abstract}

\section{Introduction}

It is broadly known that wind forcing over the surface of a stratified lake can generate basin-scale internal waves. Such long internal waves often possess amplitudes as large as ten or more meters, and their resulting current plays an important role in the transport of biogeochemical particles in lakes. From an environmental perspective it is important to study

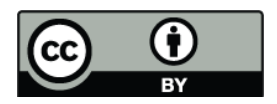

Correspondence to: T. Sakai (sakai328@gmail.com) the effects of such hydrodynamic response on trajectories of particles because of consequences relating to water quality: for instance, the nutrient-rich upwelled region, plankton clouds, local spill of chemical substances, etc., (Imberger, 1998).

Since lakes are confined bodies of water that exist in essentially isolated environments, aside from a relatively weak physical interaction with the peripheral environment through riverine inflow-outflow, wind-driven basin-scale internal waves persist in such closed domains until they perish through boundary and internal dissipation. In a large lake, in particular, internal basin response to wind forcing is comprised of Kelvin and Poncaré waves (Csanady, 1975), and these waves travel along the basin perimeter for several days, transporting particulate matter horizontally for distances comparable to the horizontal extent of the lake.

Stocker and Imberger (2003) addressed this large-scale transport phenomenon through an analytical modeling approach by employing a traditional linear hydrodynamic approximation with a circular lake model. They demonstrated the basin-scale transport of patches of Lagrangian particles that are advected by a geostrophic flow and the gravest Kelvin and Poncaré waves in a lake subject to uniform wind stresses. They also discovered that chaotic advection of particles exists, and also considered the effect of a superposed turbulent dispersion on cloud patches. The background flow model employed in their study was restricted to linear hydrostatic with homogeneous medium that perceives essentially a single vertical eigenmode, and in many examples they tracked particle paths for extensive durations, up to tens of days, under persistent wind stresses.

In practice most lakes are continuously stratified and, therefore, possess a spectrum of vertical eigenmodes which can be excited during a typical wind forcing event. Nominal wind forcing events persist for some fraction of an inertial period (a day, or perhaps two), and the consequent excited wave modes can persist for several inertial periods

Published by Copernicus Publications on behalf of the European Geosciences Union and the American Geophysical Union. 
before they have decayed substantially through dissipative processes. Furthermore, since waves of large amplitude are ubiquitous in many lakes, it is important to consider nonlinear effects of fluid acceleration in conjunction with inherent non-hydrostatic effects. Since the particle transport is dictated by the background flow, higher vertical eigenmodes plus nonlinearity of the flow may, to a greater or lesser degree, alter the fate of particles transported by the induced hydrodynamic field. The effect of the wind-stimulated, internal wave field on particle transport in closed basins is an intriguing problem, and it appears that the role of frequently excited wave modes on the characteristics of consequent transport is not very well understood.

In this contribution we extend Stoker and Imberger's novel work to consider a continuously stratified medium and, in particular, address the role of the second vertical mode within a linear-hydrostatic frame work. We also attempt to discover the effects of nonlinearity of the energetically dominant mode (i.e., the lowest vertical mode) by employing our recently developed weakly-nonlinear weakly-non-hydrostatic evolution model (Sakai and Redekopp, 2010).

\section{Continuously stratified model}

In this paper we employ a circular lake of uniform depth subject to a uniform wind stress over a non-deformable upper surface. This simple geometry eliminates surface waves plus topographic and boundary irregularities that complicate the field response. Furthermore, spatial gradients in the applied stress are excluded in this study as we seek principally to expose the role of the different modal responses on lateral particle transport. The vertical structure of the water column comprises an epilimnion of thickness $h_{1}$, a metalimnion of thickness $h_{2}$, and a hypolimninon of thickness $h_{3}$. We let both the epilimnetic and the hyplimnetic layers have the uniform densities $\rho_{1}$ and $\rho_{2}$, respectively, and set the density to vary linearly across the metalimnetic layer from $\rho_{1}$ to $\rho_{2}$. A cylindrical coordinate system $(r, \theta, z)$ is employed to describe the dynamics in the cylindrical domain, in which we position the origin of the radial coordinate at the center of the lake upper surface and set the $z$-axis perpendicular to the upper surface with a direction pointing upward from the lake surface. A similar model is given in Csanady (1972) with application to the North American Great Lakes. In what follows we choose to describe briefly the model formulation, both for clarification and to have the presentation self-contained.

The uniform wind stress is oriented in a particular direction, say the positive $x$-direction, as given by $\boldsymbol{\tau}=\tau(z, t) \mathbf{e}_{x}$, and the wind stress is applied for a finite time $0 \leq t<t_{0}$. We assume that the wind stress function $\boldsymbol{\tau}(z, t)$ is separable in space and time, and that the stress decreases linearly with depth across the eplimnion and vanishes at the top of the metaliminon (e.g., see Monismith, 1987; note that the final result is independent of the shape of the stress function so long as the stress vanishes at the top of the metalimnion; see also Sakai and Redekopp, 2009a). The analytical form chosen for $\tau(z, t)$ for $-h_{1}<z \leq 0$ is given as

$\tau(z, t)=\rho_{0} u_{* 0}^{2}\left\{1-u_{\mathrm{S}}\left(t-t_{0}\right)\right\} \frac{z+h_{1}}{h_{1}}$,

where $\rho_{0}$ is a reference density, $u_{* 0}$ is a friction velocity and $u_{\mathrm{s}}(t)$ is the Heaviside step function.

The field equations consist of the conservation of mass constraint for an incompressible fluid, the linearized, inviscid momentum equations subject to the applied wind stress under the hydrostatic and Boussinesq approximations, plus the linearized continuity equation expressing the conservation of the density of a fluid parcel in its motion. These equations, respectively, have the forms:

$$
\begin{aligned}
& \frac{\partial u}{\partial r}+\frac{u}{r}+\frac{1}{r} \frac{\partial v}{\partial \theta}+\frac{\partial w}{\partial z}=0, \\
& \frac{\partial u}{\partial t}-f v=-\frac{1}{\rho_{0}} \frac{\partial p}{\partial r}+\frac{1}{\rho_{0}} \frac{\partial \tau}{\partial z} \cos \theta, \\
& \frac{\partial v}{\partial t}+f u=-\frac{1}{\rho_{0} r} \frac{\partial p}{\partial \theta}-\frac{1}{\rho_{0}} \frac{\partial \tau}{\partial z} \sin \theta, \\
& 0=-\frac{1}{\rho_{0}} \frac{\partial p}{\partial z}-\sigma, \\
& \frac{\partial \sigma}{\partial t}=N^{2}(z) w,
\end{aligned}
$$

The velocity components $(u, v, w)$ correspond to the velocity in the radial, azimuthal and vertical directions; $f$ is an inertial frequency; $p$ is a perturbation pressure; $\sigma$ is a perturbation buoyancy defined by $\sigma=\rho g / \rho_{0}$, where $\rho$ is the perturbation buoyancy and $g$ is a gravitational constant; and $N^{2}(z)$ is the Brunt-Väisälä frequency defined by $N^{2}(z)=$ $-\rho_{\mathrm{S}}^{\prime}(z) g / \rho_{0}$, where $\rho_{\mathrm{s}}(z)$ is a static (stable) density distribution. Contribution of the wind stress is accounted in the horizontal momentum equations. Since Eq. (2) does not take account of any energy damping and nonlinearity, the analytical model is limited to representing the internal dynamics only for time periods, at most, on the order of several days (or inertial periods) (Csanady, 1968).

The field variables are projected rationally in terms of vertical eigenfunctions according to the relations

$$
\begin{aligned}
& \left(u, v, p / \rho_{0}\right)=\sum_{n}(U, V, P)_{n}(r, \theta, t) \phi_{n}^{\prime}(z), \\
& w=\sum_{n} W_{n}(r, \theta, t) \phi_{n}(z), \\
& \sigma=\sum_{n} Z_{n}(r, \theta, t) N^{2}(z) \phi_{n}(z) .
\end{aligned}
$$

The field variables in upper case symbols represent the amplitudes of respective vertical eigenmodes, and $\phi_{n}(z)$ is the vertical eigenfunction defined by the boundary value problem 
$\phi_{n}^{\prime \prime}+\frac{N^{2}(z)}{c_{n}^{2}} \phi_{n}=0, \quad \phi_{n}=0$ at $z=0$ and $z=-H$,

where $H$ is a total lake depth as given by $H=h_{1}+h_{2}+h_{3}$. The eigenvalue $c_{n}$ is the propagation speed of a given vertical eigenmode in the longwave limit, and $\phi_{n}$ is normalized by its maximum value. Analytical expressions of eigenpairs in the present formulation are available, but are not given here for brevity of presentation. The orthogonality relation of the eigenfunction is given by

$\int_{-H}^{0} \phi_{m}^{\prime} \phi_{n}^{\prime} \mathrm{d} z=\frac{c_{n}^{2}}{\mu_{n}} \delta_{m n}$, and $\mu_{n}=\int_{-H}^{0} N^{2} \phi_{n}^{2} \mathrm{~d} z$,

where $\delta_{m n}$ is the Kronecker delta.

In order to study parametric dependencies on particle dynamics, we scale the problem using $r_{0}$, the lake radius, as a horizontal length scale, the epilimnion depth $h_{1}$ as the vertical length scale, and $N_{0}^{-1}$ as a time scale $\left(N_{0}^{2}=\left(\rho_{2}-\right.\right.$ $\left.\left.\rho_{1}\right) g / \rho_{0} h_{2}\right)$. Specifically, $t$ is scaled by $r_{0} / N_{0} h_{1} ; c_{n}$ and $W_{n}$ are scaled by $N_{0} h_{1} ;(U, V, P)_{n}$ are scaled by $N_{0} h_{1}^{2}$; and $Z_{n}$ is scaled by $h_{1}$. Employing scaled variables, and substituting Eqs. (1) and (3) into Eq. (2) and using Eq. (5), we obtain an evolution set for any given vertical eigenmode $(n=1,2, \ldots)$ in the following dimensionless form:

$$
\begin{aligned}
& \frac{\partial U_{n}}{\partial t}-\mathcal{B} V_{n}+c_{n}^{2} \frac{\partial Z_{n}}{\partial r}=\frac{k_{n}}{\mathcal{W}}\left[1-u_{\mathrm{s}}\left(t-t_{0}\right)\right] \cos \theta, \\
& \frac{\partial V_{n}}{\partial t}+\mathcal{B} U_{n}+c_{n}^{2} \frac{1}{r} \frac{\partial Z_{n}}{\partial \theta}=-\frac{k_{n}}{\mathcal{W}}\left[1-u_{\mathrm{s}}\left(t-t_{0}\right)\right] \sin \theta, \\
& \frac{\partial Z_{n}}{\partial t}+\frac{\partial U_{n}}{\partial r}+\frac{U_{n}}{r}+\frac{1}{r} \frac{\partial V_{n}}{\partial \theta}=0,
\end{aligned}
$$

In the first two equations the parameter $k_{n}=c_{n}^{2} \phi_{n}^{\prime}(0) / \mu_{n}$, and the Burger number $\mathcal{B}$ and the Wedderburn number $\mathcal{W}$ are defined by

$\mathcal{B}=\frac{f r_{0}}{N_{0} h_{1}}=\frac{r_{0}}{\left(N_{0} h_{1}\right) / f} \quad$ and $\mathcal{W}=\frac{N_{0}^{2} h_{1}^{3}}{u_{* 0}^{2} r_{0}}$.

The Burger number, in this formulation, is a lake radius relative to the internal Rossby radius, measuring the effect of earth's rotation. The inverse of the Wedderburn number measures the wind stress strength relative to the counter-acting baroclinic pressure gradient. According to laboratory experiments, internal responses are dominated by internal gravity waves for values of the Wedderburn number in the range $1<\mathcal{W}<5$ (Horn et al., 2001).

We note at this point that Eq. (6) has a similar structure as the set corresponding to the single-eigenmode model (Csanady, 1968; Stocker and Imberger, 2003). The solution for a single vertical eigenmode in our case can be obtained analytically through the Laplace transform method (see Csanady, 1968 and Stocker and Imberger, 2003 for detail) and expressed as:

$$
\begin{aligned}
U_{n}(r, \theta, t) & =-\frac{k_{n}}{\mathcal{W B}}\left\{1-\frac{1}{r} \frac{I_{1}\left(\alpha_{0 n} r\right)}{I_{1}\left(\alpha_{0 n}\right)}\right\}\left\{1-u_{\mathrm{s}}\left(t-t_{0}\right)\right\} \sin \theta \\
& -\frac{k_{n}}{\mathcal{W} \mathcal{B}} \sum_{m=1}^{\infty} \frac{A_{m n}}{1-\omega_{m n}^{2}}\left\{\omega_{m n} \frac{\alpha_{m n} R_{1}^{\prime}\left(\alpha_{m n} r\right)}{R_{1}\left(\alpha_{m n}\right)}\right. \\
& \left.-\frac{1}{r} \frac{R_{1}\left(\alpha_{m n} r\right)}{R_{1}\left(\alpha_{m n}\right)}\right\}\left\{\sin \left(\theta-\mathcal{B} \omega_{m n} t\right)\right. \\
& \left.-u_{\mathrm{s}}\left(t-t_{0}\right) \sin \left(\theta-\mathcal{B} \omega_{m n}\left(t-t_{0}\right)\right)\right\}, \\
V_{n}(r, \theta, t) & =-\frac{k_{n}}{\mathcal{W B}}\left\{1-\alpha_{0 n} \frac{I_{1}^{\prime}\left(\alpha_{0 n} r\right)}{I_{1}\left(\alpha_{0 n}\right)}\right\}\left\{1-u_{\mathrm{s}}\left(t-t_{0}\right)\right\} \cos \theta \\
& +\frac{k_{n}}{\mathcal{W B}} \sum_{m=1}^{\infty} \frac{A_{m n}}{1-\omega_{m n}^{2}}\left\{\alpha_{m n} \frac{R_{1}^{\prime}\left(\alpha_{m n} r\right)}{R_{1}\left(\alpha_{m n}\right)}\right. \\
& \left.-\frac{\omega_{m n}}{r} \frac{R_{1}\left(\alpha_{m n} r\right)}{R_{1}\left(\alpha_{m n}\right)}\right\}\left\{\cos \left(\theta-\mathcal{B} \omega_{m n} t\right)\right. \\
& \left.-u_{\mathrm{s}}\left(t-t_{0}\right) \cos \left(\theta-\mathcal{B} \omega_{m n}\left(t-t_{0}\right)\right)\right\}, \\
Z_{n}(r, \theta, t) & =\frac{k_{n}}{\mathcal{W} c_{n}^{2}} \frac{I_{1}\left(\alpha_{0 n} r\right)}{I_{1}\left(\alpha_{0 n}\right)}\left\{1-u_{\mathrm{s}}\left(t-t_{0}\right)\right\} \cos \theta \\
& +\frac{k_{n}}{\mathcal{W} c_{n}^{2}} \sum_{m=1}^{\infty} A_{m n} \frac{R_{1}\left(\alpha_{m n} r\right)}{R_{1}\left(\alpha_{m n}\right)}\left\{\cos \left(\theta-\mathcal{B} \omega_{m n} t\right)\right. \\
& \left.-u_{\mathrm{s}}\left(t-t_{0}\right) \cos \left(\theta-\mathcal{B} \omega_{m n}\left(t-t_{0}\right)\right)\right\},
\end{aligned}
$$

Parameters appearing in these expressions are defined as

$$
\begin{aligned}
& \alpha_{0 n}=\frac{\mathcal{B}}{c_{n}}, \quad \alpha_{m n}=\frac{\mathcal{B}}{c_{n}} \sqrt{\left|\omega_{m n}^{2}-1\right|}, \\
& \text { and } A_{m n}=\frac{\omega_{m n}-1}{1+\omega_{m n}-\left(\mathcal{B} / c_{n}\right)^{2} \omega_{m n}^{3}},
\end{aligned}
$$

and $R_{1}\left(\alpha_{m n} r\right)$ is a radial eigenfunction which is either the Bessel function $J(x)$ or the modified-Bessel function $I(x)$ of order one depending on the value of the eigenfrequency $\omega_{m n}$ :

$R_{1}\left(\alpha_{m n} r\right)=\left\{\begin{array}{l}I_{1}\left(\alpha_{m n} r\right), \omega_{m n}^{2}<1, \\ J_{1}\left(\alpha_{m n} r\right), \omega_{m n}^{2}>1 .\end{array}\right.$

The modal solution $I_{1}(x)$ is associated with the Kelvin wave (subinertial) solution, and that associated with $J_{1}(x)$ is the Poincaré wave (superinertial) solution. The eigenfrequency is obtained from the characteristic equation

$R_{1}\left(\alpha_{m n}\right)-\omega_{m n} \alpha_{m n} R_{1}^{\prime}\left(\alpha_{m n}\right)=0$.

Considering a basin in the northern hemisphere, a radial eigenmode with a positive eigenfrequency travels in a cyclonic direction and an eigenmode with a negative eigenfrequency travels in an anti-cyclonic direction. The gravest radial mode in cyclonic wave modes is the Kelvin wave, and the anti-cyclonic counterpart is the Poincare wave. There exist infinitely many Poincaré wave modes with both cyclocnic and anti-cyclonic polarities, but only one Kelvin wave 


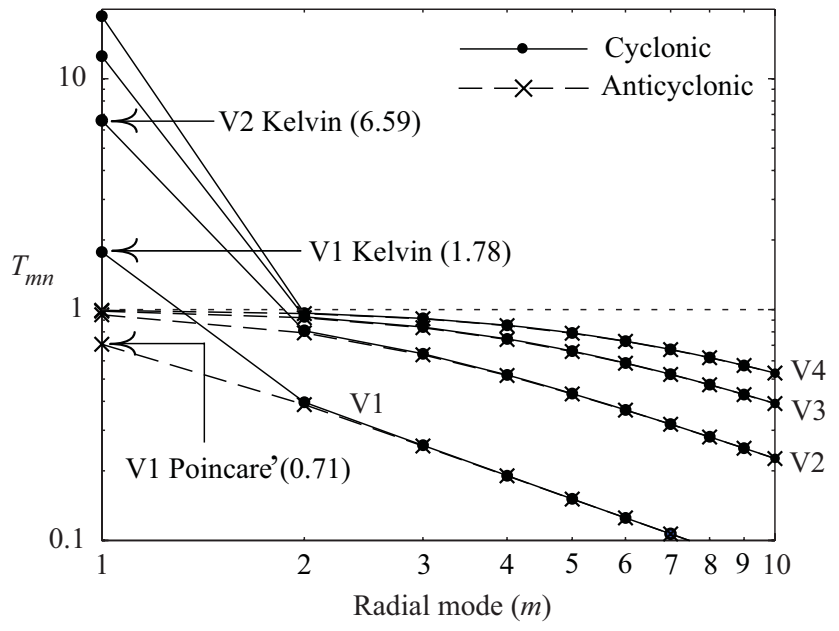

Fig. 1. Fundamental periods normalized by the inertial period $T_{i}$ for $\mathcal{B}=2, h_{2}=1$ and $h_{3}=2$.

mode exists in the entire radial eigenspace for given vertical mode. Internal current profiles of the Kelvin and the Poincare waves are completely opposite. The Kelvin waves generate the largest along-shore current at the basin perimeter and the current decays exponentially toward the basin center. The Poincare waves, on the other hand, generate the largest offshore current at the basin center and the current decreases to zero at the basin perimeter. The first grouped term in Eq. (8) in each modal solution represents the flow component that is geostrophically balanced with the applied wind stress. Since the curl of the uniform wind stress is zero, this geostrophic flow entirely vanishes immediately after the wind stress is turned off. The field response following the wind event is the linear combination of the Kelvin wave mode and a family of the Poincaré wave modes. It has been reported that the geostrophic flows persistent in the field (e.g., gyric flows generated from non-uniform wind stresses, topographic variations, prolonged wind forcing, etc.) contribute to a significant fraction of the dispersion rate of a particle cloud (Patil et al., 2010; Stocker and Imberger, 2003), but such flow conditions are excluded in the present study.

Although not shown here, the solution Eq. (8) can be easily extended to the solution with arbitrary temporal forcing by discretizing the temporal forcing function into pieces of single-window functions, and then performing a linear superposition of their corresponding solutions.

\section{Energetically dominant eigenmodes}

It is important to note that in a continuously stratified domain each vertical eigenmode possesses its own horizontal (radial only, in this study) eigenspace, and a spectrum of verticalhorizontal eigenmodes coexist in the domain. In order to gain

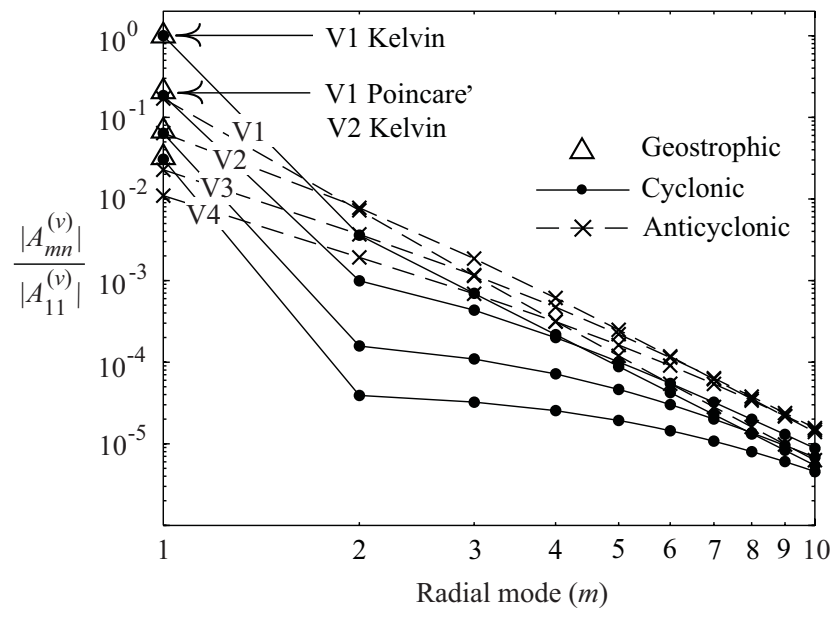

Fig. 2. Modal velocity amplitudes for $\mathcal{B}=2, h_{2}=1$ and $h_{3}=2$. All values are normalized by the value of the Kelvin wave of vertical mode-one $A_{11}^{(v)}$. Amplitudes of geostrophic waves are plotted on radial mode-one as reference values.

some insight to the relative time scale of these modes, the fundamental periods $T_{m n}\left(=2 \pi / \mathcal{B} \omega_{m n}\right)$ of the first four vertical modes are computed as functions of the radial mode for the case of $\mathcal{B}=2, h_{2}=1$ and $h_{3}=2$ (Fig. 1). The fundamental periods are normalized by the inertial period $T_{i}(=2 \pi / \mathcal{B})$. In this example the fundamental period of the Kelvin wave of the first vertical mode (V1) is about twice as long as the inertial period, but the Kelvin wave of the second vertical mode (V2) is much slower and the fundamental period is about seven times as long as the inertial period. The relative differences of the fundamental periods of the Kelvin wave modes of different vertical modes are remarkably distinct, but the Poincaré waves of the lowest radial mode have similar time scales that are near the inertial period. For radial modes larger than one, the cyclonic and anti-cyclonic Poincaré wave modes exist in pairs and their frequencies are nearly identical.

Figure 2 shows the corresponding amplitudes of the fundamental wave modes presented in Fig. 1. Although there can be several ways to define the modal amplitudes, we particularly refer to the velocity amplitudes $\left(U_{n}, V_{n}\right)$ in Eq. (8) and simply extract a mode-dependent scaling factor from each modal component and refer to the factor as the modal velocity amplitude $A_{m n}^{(v)}$ as given by

$A_{m n}^{(v)}=\frac{k_{n} A_{m n}}{1-\omega_{m n}^{2}}$.

For a geostrophic component the corresponding amplitude is defined as $A_{n}^{(G)}=k_{n}$. From Fig. 2 the Kelvin waves of the lowest two vertical modes and the Poincare wave of the lowest vertical mode have the most significant amplitudes, and the amplitudes of all the other modes are one or more orders of magnitude smaller. Geostrophic components have 


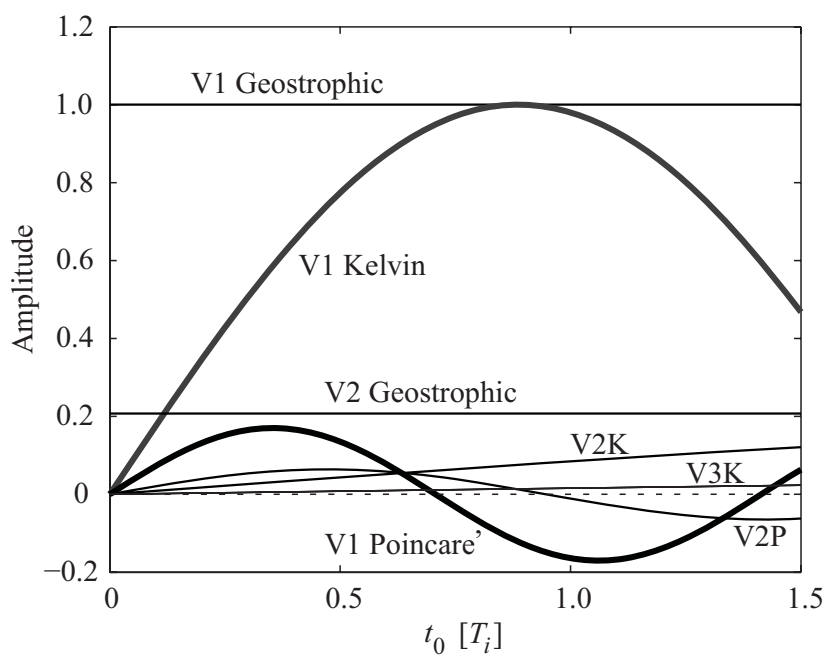

Fig. 3. Several modal velocity amplitudes as functions of forcing time $t_{0}$. All values are normalized by the maximum value of the amplitude of the Kelvin wave of vertical mode-one. Amplitudes of geostrophic waves (effective during wind event only) are included as references.

large amplitudes similar to those of the Kelvin waves, but the geostrophic flows exist only during the wind forcing event as mentioned earlier. In the field solution expressions (Eq. 8), trigonometric terms with the step function $u_{\mathrm{s}}$ are the "reaction" terms arising from the cessation of wind forcing after a finite time $\left(t=t_{0}\right)$. After the wind event these reaction terms result in the extra factor $2 \sin \left(\mathcal{B} \omega_{m n} t_{0} / 2\right)$ that permanently scales the wave amplitude following the wind cessation.

Modal velocity amplitudes of several significant wave modes are computed as functions of the wind forcing duration $t_{0}$ and shown in Fig. 3 . These results particularly correspond to the case presented in Figs. 1 and 2, but one can obtain similar pictures for different parameter sets. It can be observed from the figure that, if the wind event is within or comparable to one inertial period, the V1 Kelvin wave is energetically dominant (i.e., the amplitude is the largest) followed by the V1 Poincaré wave, and that waves of vertical mode two (or higher) are energetically insignificant relative to the dominant V1 Kelvin-Poincaré wave pair. Therefore, from an "energetics" point of view, considering only the V1 Kelvin-Poincaré pair can be a reasonable choice. In fact, a number of previous studies considered the dynamics involving only a single (the lowest) vertical mode; for instance, by exploiting the traditional two-layer stratification model rather than one with a metaliminion with finite thickness and continuous stratification.

\section{Effects of the second vertical mode on particle transport}

Physical space coordinates $(r, \theta, z)$ of a Lagarangian particle are dictated by the following set of fully-coupled nonlinear ordinary differential equations:

$$
\begin{aligned}
\frac{\mathrm{d} r}{\mathrm{~d} t} & =\sum_{n} U_{n}(r, \theta, t) \phi_{n}^{\prime}(z), \\
\frac{\mathrm{d} \theta}{\mathrm{d} t} & =\sum_{n} \frac{1}{r} V_{n}(r, \theta, t) \phi_{n}^{\prime}(z), \\
\frac{\mathrm{d} z}{\mathrm{~d} t} & =-\sum_{n}\left(\frac{\partial U_{n}}{\partial r}+\frac{U_{n}}{r}+\frac{1}{r} \frac{\partial V_{n}}{\partial \theta}\right) \phi_{n}(z) .
\end{aligned}
$$

In this paper we are particularly interested in the evolution of a localized cloud (patch) of particles over the lake top or bottom surfaces, such as the transport of a local cloud of phytoplankton bloom near a beach, the transport of chemically polluted regions condensed at the bottom of a lake, etc. Although the particle transport is three-dimensional in nature, over the top and the bottom surfaces of the present lake model the particle transport is in-plane with a use of nondeformable boundary conditions. For the background velocity field we consider only the first few energetically dominant flow components, which are the gravest Kelvin wave modes, and the gravest Poincaré wave modes, plus the geostrophic flow components of the lowest two vertical modes (V1 and V2). Inclusion of the higher eigenmodes does not alter the results dramatically. Analytical solutions (Eq. 8) are exploited to directly compute the flow field, and the set of tracer equations (Eq. 13) is integrated forward-in-time for several inertial periods by using the Runge-Kutta method of fourth order. For all cases presented here the lake is subject to wind stresses corresponding to a Wedderburn number of unit order, and the wind stresses are imposed for some fraction of an inertial period during a single wind event.

Figure 4 exhibits the snap shots of four different cloud patches taken at every inertial period for the first four inertial periods, with time increasing along a downward progression in a particular column in the figure. The lake is subject to a uniform wind stress of $\mathcal{W}=1$ directed from the left to the right (as looking down the lake) for the first one third of the inertial period, and the stress is permanently turned off thereafter. Particle clouds having two concentric radii (the outer radius is $0.7 r_{0}$ and the inner radius is $0.35 r_{0}$ ) are initially centered at four extreme sides of the top surface of the lake (i.e., the windward and the leeward ends, plus the left and the right sides, all relative to the positive direction of the wind vector). The physical parameters for the patch-tracking calculations are $\mathcal{B}=2, h_{2}=1$ and $h_{3}=2$. It can be seen from Fig. 4 that the cloud patch initially set at the right side of the wind vector (third column) stretches in along-shore directions as it is advected in a cyclonic direction. It should be noted that the cloud elongates quite remarkably, roughly by 
(a)
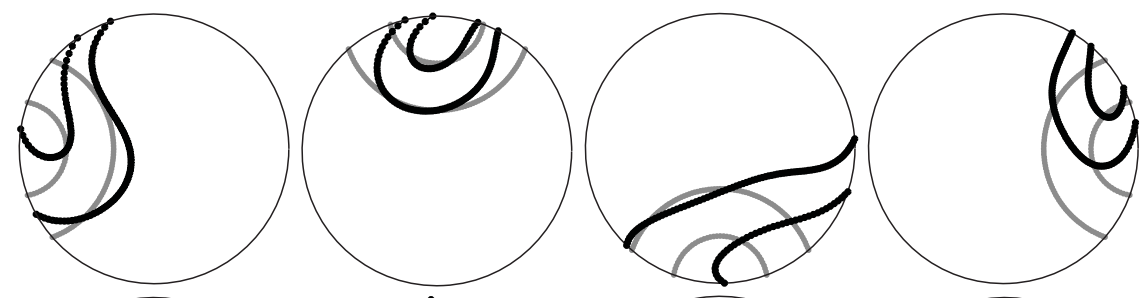

(b)

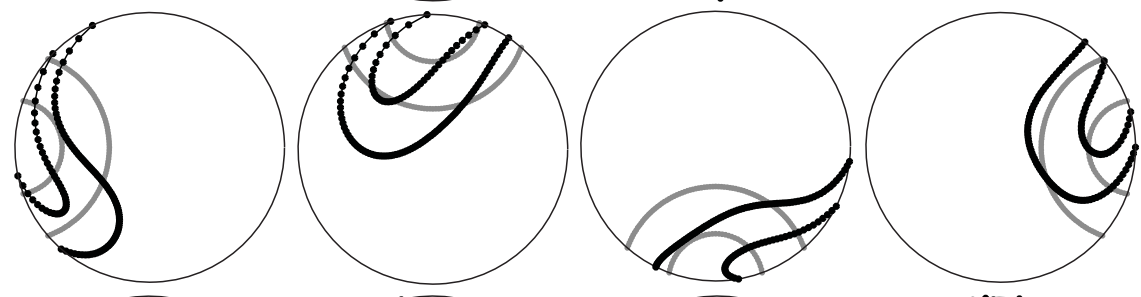

(c)
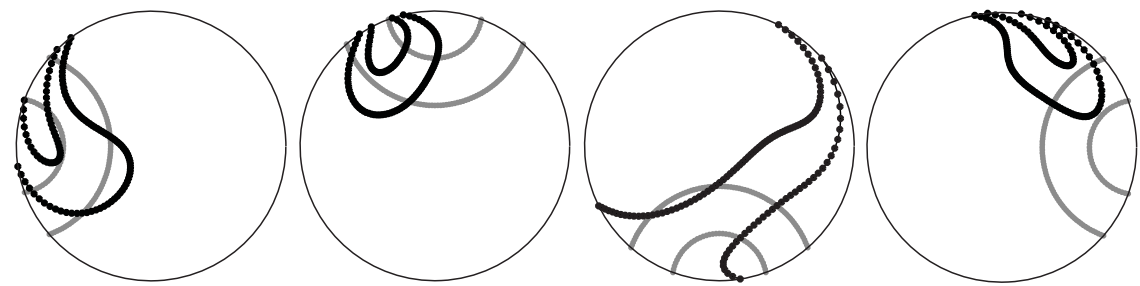

(d)

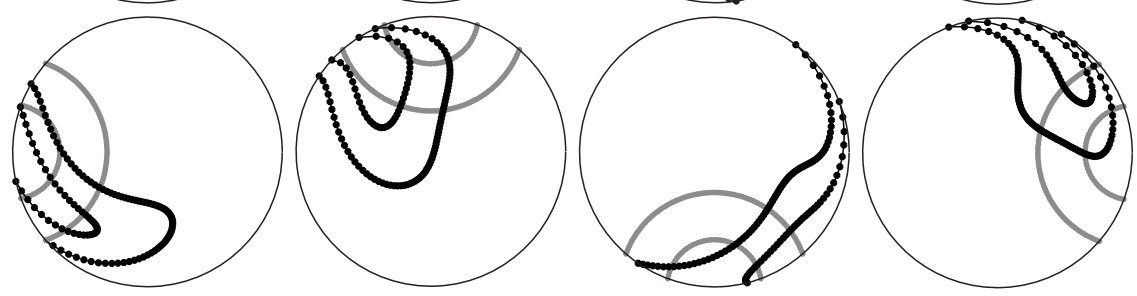

Fig. 4. Snap shots of several cloud patches at $t=T_{i}$ (row-a), $t=2 T_{i}$ (row-b), $t=3 T_{i}$ (row-c) and $t=4 T_{i}$ (row-d). Grayed points represent the initial position of particles. A uniform wind stress of $\mathcal{W}=1$ is applied for $0<t<T_{i} / 3$ from the left to the right. The background velocity field contains the geostrophic flows (during only the wind event) and the Kelvin and the Poincare wave modes of the lowest two vertical modes. $\left(\mathcal{B}=2, h_{2}=1, h_{3}=2\right)$.

a factor of three, and that the frontal edge of the cloud travels for a distance comparable to the lake diameter after three inertial periods. The cloud on the opposite side (i.e., the left side) of the wind vector (second column) squeezes in alongshore directions as it travels in a cyclonic direction, and the cloud protrudes significantly in an off-shore direction and recedes in an on-shore direction oscillatory in time. The cloud patch initially at the leeward end (fourth column) is skewed in along-shore directions with pronounced along-shore transport adjacent to the basin perimeter, and the cloud as a whole is transported in a cyclonic direction. The cloud patch initially at the windward end (first column) is also skewed similarly, but with less remarkable transport of the near-shore portion when compared to the near-shore transport of clouds at other positions around the perimeter of the lake.

In order to quantify the effects of the second vertical mode, flow components of the second vertical mode are all turned off and the resulting V1-only transport is compared with the original V1+V2 transport. Differences in these mode-driven transports depend on the initial position of a cloud patch relative to the wind direction. We find that the transport is most remarkably enhanced by V2-driven current when the cloud is initially positioned to the right of the wind vector. In what follows, therefore, our presentation focuses exclusively on this particular case. Snap shots of the cloud patch evolving in the V1-only field are compared in Fig. 5 with those corresponding to in the $\mathrm{V} 1+\mathrm{V} 2$ field previously presented in Fig. 4. It can be seen in Fig. 5 that, as time increases, the transport of particles near the basin perimeter is remarkably increased for the $\mathrm{V} 1+\mathrm{V} 2$ field relative to that occurring under the V1-only field. However, the off-shore protrusions of the clouds in these two fields appear to be very similar. This is also true regardless of initial location of the cloud.

To quantify somewhat the development of an evolving cloud patch with a given initial radius of $r_{0} / 2$, the gross dimensions are measured as functions of time under both the V1-only and the V1+V2 velocity fields. The particular cloud dimensions which are tracked include the alongshore (cyclonic) displacement of both the front and the tail vertices of the cloud position along the basin perimeter, the maximum off-shore protrusion into the lake, and the total area of the cloud. The temporal development of these 
(a)

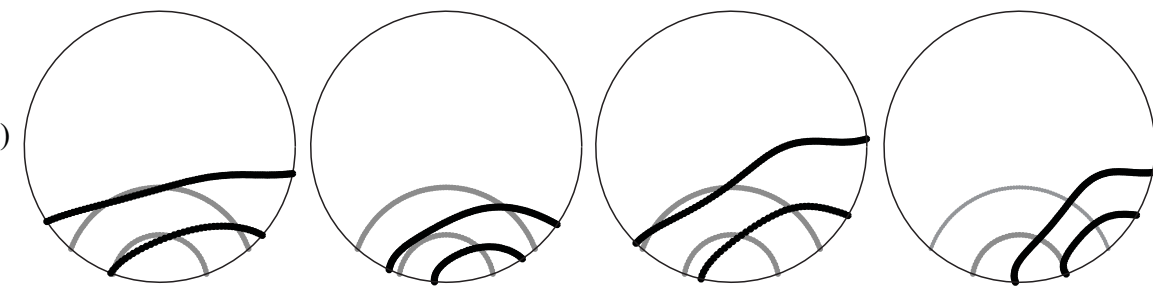

(b)

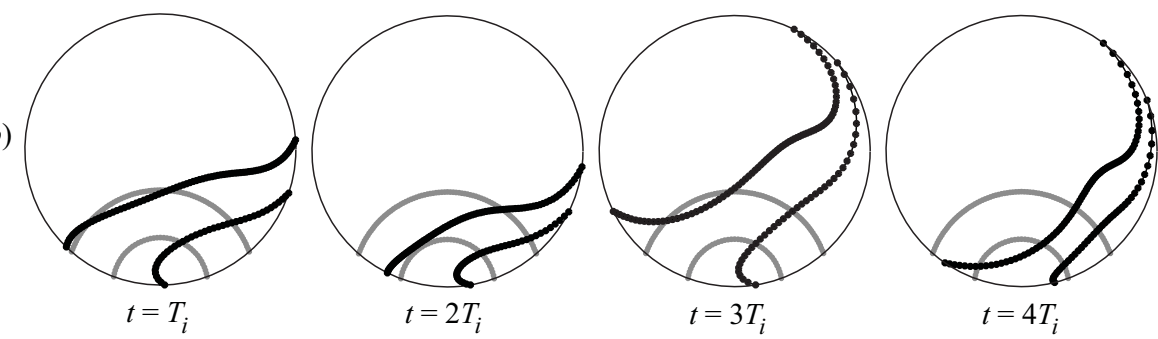

Fig. 5. Comparison of cloud patch evolutions obtained in the field containing only the first vertical mode (row-a) and in the field including the second vertical mode (row-b). Grayed points represent the initial position of particles. $\left(\mathcal{B}=2, h_{2}=1, h_{3}=2, \mathcal{W}=1\right)$.
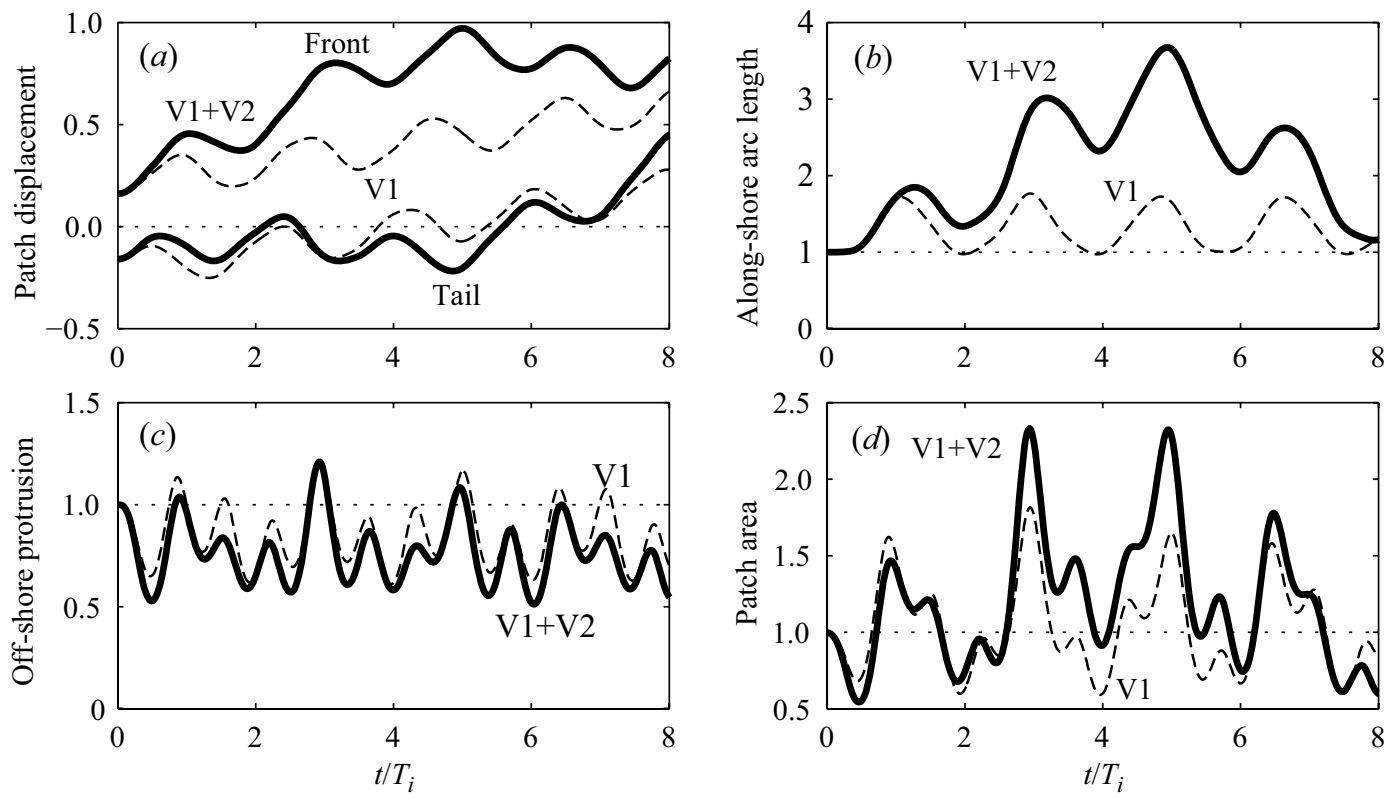

Fig. 6. Time series of (a) along-shore displacement of cloud front and tail vertices, (b) along-shore arch length of the cloud on the basin perimeter side, (c) off-shore protrusion of the cloud, and (d) cloud patch area, obtained in V1-only field and V1+V2 field. A cloud patch of radius $r_{0} / 2$ is initially placed at the right end of the basin relative to the positive wind vector. The along-shore displacement (a) is normalized by $\pi r_{0}$, the along-shore length (b) and the patch area (c) are normalized by their respective initial values, and the off-shore protrusion (d) is normalized by their initial radius of the cloud patch. $\left(\mathcal{B}=2, h_{2}=1, h_{3}=2, \mathcal{W}=1\right)$.

quantitative measures is presented in Fig. 6. It is clearly observed from the figure that the along-shore cloud displacement and length are doubled when the field contains the second vertical mode. The along-shore dimensions fluctuate approximately with the V1 Kelvin wave period $\left(=1.7 T_{i}\right)$, and in the case of the V1+V2 field the along-shore length of the cloud is modulated by a lower frequency component which is nearly equal to the V2 Kelvin wave period $\left(=6.6 T_{i}\right)$. At a fixed position along the basin boundary, the current induced by the Kelvin wave is in a cyclonic direction during the half Kelvin wave period, but reverses to an anti-cyclonic direction for the following half-Kelvin-wave period. Such reversals in flow direction underlie the oscillatory behavior exhibited in the along-shore dimensions. A resultant persistent transport in the cyclonic direction arises due to the nonlinear (Stokes) drift. The maximum field velocity associated with the V1 
Kelvin wave is 0.34 dimensionless units while the V2 Kelvin wave counterpart is 0.12 , with the maxima in both cases occurring at the basin perimeter. Although the V2 Kelvin wave induces a considerably smaller peak velocity, the V2 Kelvin wave can transport particles near the basin boundary for a distance comparable with the V1-only transport. This enhanced transport relative to the peak velocity is due to the fact that particle transport is given by the time-integral of the velocity at the position of the particle. The disparately slow V2 Kelvin wave is resident in the same region of the basin for a considerably long time and, thus, the resultant particle transport becomes non-negligible.

The off-shore protrusion, on the contrary, fluctuates much faster with periods near the inertial period, and the off-shore protrusion in the V1+V2 field differs from that in the V1only field only slightly. Since the Poincare waves induce the largest off-shore current across the center of the lake, the off-shore current can push and pull the cloud in radial directions as frequent as rational multiples of their fundamental periods which are near the inertial period. The area of the cloud patch also fluctuates in time because the horizontal divergence of the flow is non-vanishing, and is balanced by the rate of change of vertical flows (driven by internal waves) as dictated by the conservation of mass constraint. The patch area for the $\mathrm{V} 1+\mathrm{V} 2$ field is roughly doubled after several inertial periods owing to the aforementioned enhanced alongshore stretching by the V2 Kelvin wave.

In order to confirm the effects of internal waves on the evolution of the cloud shape, frequency spectra of the alongshore cloud length and the off-shore cloud protrusion are computed for different Burger numbers $(\mathcal{B}=1,2$ and 4$)$. These spectra are presented in Fig. 7, where it is clearly seen that in all cases the Kelvin waves comprise the dominant component in the time signal of the along-shore arc length. Further, the signal amplitude associated with the V2 Kelvin wave is of the same order as that of the V1 Kelvin wave. It is also evident from the figure that the time signal of the off-shore protrusion is dominated by the frequencies of the Poincaré waves and their higher harmonics. There is one exception in the result for $\mathcal{B}=1$ (Fig. 7d) where the V1 Kelvin wave is also among the dominant components contributing in the off-shore protrusion. This is because, for a small lake $\left(B / c_{1}<\sqrt{2}\right)$, the V1 Kelvin does not decay exponentially in the off-shore direction. As such, the associated current in the basin center serves as the off-shore current.

It should be added that, since the cloud patch is moving, the time signals of the measured dimensions do not manifest exactly the same frequency components as the analytical eigenfrequencies due to the Doppler effect. To obtain reasonable results as presented in Fig. 7, the drift rate of the cloud is diminished by reducing the wind stress strength (i.e., use of larger Wedderburn numbers), and the cloud dimensions were again sampled for a long time in order to capture the small frequency components (i.e., the Kelvin waves) without aliasing.
All the results presented so far pertain to particle transport over the top surface of the lake. Although not presented here, we also considered the transport over the lake bottom surface. Similar trends as seen in the forgoing figures have been found for bottom transport. However, since in many lakes the hypolimnion is usually deeper than the epilimnion, the horizontal current is far smaller in the hypolimnion than in the epilimnion, resulting in a much diminished transport over the bottom surface.

\section{Nonlinearity of the background flow}

Recent studies of nonlinear internal wave evolutions revealed the generation of solitary waves from a steepened Kelvin wave and the pseudo-recurrence character of Poincaré waves (Sakai and Redekopp, 2010; de la Fuente et al., 2008). The remainder of this report is motivated by an interest as to how nonlinear effects modify the evolution of a particle cloud relative to that resulting under a linearized hydrodynamic description. To simplify the problem we restrict the consideration to self-nonlinear effects of the energetically dominant, lowest vertical mode and keep the second vertical mode linear hydrostatic. This simplification neglects not only the self-nonlinearity of the second vertical mode but also the inter-modal nonlinear interactions. For the flow field model describing the first vertical mode, we employ a weaklynonlinear, weakly-nonhydrostatic version of the modal evolution equations (Eq. 6) as given by:

$$
\begin{gathered}
\frac{\partial U_{1}}{\partial t}-\mathcal{B} V_{1}+c_{1}^{2} \frac{\partial Z_{1}}{\partial r}=-\left\{\alpha \left(U_{1} \frac{\partial U_{1}}{\partial r}+\frac{V_{1}}{r} \frac{\partial U_{1}}{\partial \theta}\right.\right. \\
\left.\left.-\frac{V_{1}^{2}}{r}\right)+\beta D_{1} U_{1}\right\}+\Lambda^{2} \gamma \frac{\partial}{\partial t}\left(\frac{\partial D_{1}}{\partial r}\right), \\
\frac{\partial V_{1}}{\partial t}+\mathcal{B} U_{1}+c_{1}^{2} \frac{1}{r} \frac{\partial Z_{1}}{\partial \theta}=-\left\{\alpha \left(U_{1} \frac{\partial V_{1}}{\partial r}+\frac{V_{1}}{r} \frac{\partial V_{1}}{\partial \theta}\right.\right. \\
\left.\left.+\frac{U_{1} V_{1}}{r}\right)+\beta D_{1} U_{1}\right\}+\Lambda^{2} \gamma \frac{\partial}{\partial t}\left(\frac{1}{r} \frac{\partial D_{1}}{\partial \theta}\right), \\
\frac{\partial Z_{1}}{\partial t}+\frac{\partial U_{1}}{\partial r}+\frac{U_{1}}{r}+\frac{1}{r} \frac{\partial V_{1}}{\partial \theta}= \\
-\left\{\beta\left(U_{1} \frac{\partial Z_{1}}{\partial r}+\frac{V_{1}}{r} \frac{\partial Z_{1}}{\partial \theta}\right)+\alpha D_{1} Z_{1}\right\} .
\end{gathered}
$$

In these equations the symbol $D_{1}$ denotes the horizontal divergence given by $D_{1}=\partial U_{1} / \partial r+U_{1} / r+\partial V_{1} / r \partial \theta$; the parameter $\Lambda$ is a depth-to-radius aspect ratio as given by $\Lambda=h_{1} / r_{0}$; and, the parameters $\alpha, \beta$ and $\gamma$ are integral coefficients containing the essence of the vertical structure as defined by 

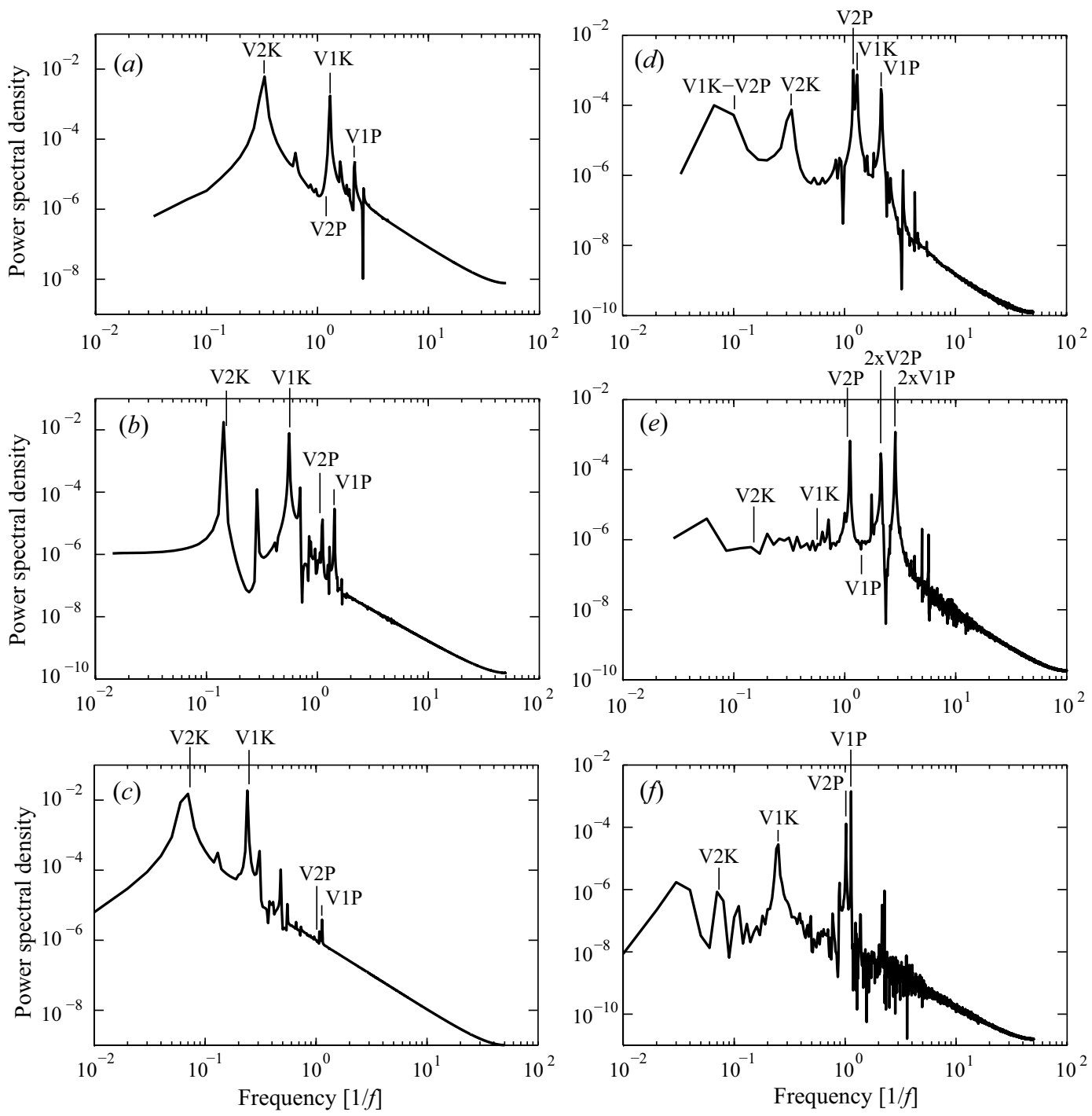

Fig. 7. Frequency spectrum of along-shore arc length $(\mathbf{a}, \mathbf{b}, \mathbf{c})$ and off-shore protrusion $(\mathbf{d}, \mathbf{e}, \mathbf{f})$ of a cloud patch of radius $r_{0} / 2$ initially placed at the right end of the basin relative to the positive wind vector for $\mathcal{B}=1$ with $\mathcal{W}=4(\mathbf{a}, \mathbf{d}), \mathcal{B}=2$ with $\mathcal{W}=2(\mathbf{b}, \mathbf{e})$ and $\mathcal{B}=4$ with $\mathcal{W}=1(\mathbf{c}, \mathbf{f})$. Frequencies of fundamental wave modes are also marked in the figure and denoted as "V1K"(=V1 Kelvin), "V2K"(=V2 Kelvin), "V1P"(=V1 Poincaré) and "V2P"(=V2 Poincaré). On panel (d) "V1K-V2P" represents the difference frequency between V1K and V2P wave modes. On panel (e) " $2 \times$ " represents the double-harmonic frequency. ( $h_{2}=1$ and $h_{3}=2$ for all cases).

$\alpha=\frac{c_{1}^{2}}{\mu_{1}} \int_{-H}^{0}\left(\phi_{1}^{\prime}\right)^{3} \mathrm{~d} z, \quad \beta=\frac{1}{\mu_{1}} \int_{-H}^{0} \phi_{1}^{\prime} \phi_{1}^{2} N^{2} \mathrm{~d} z$

and $\gamma=\frac{c_{1}^{2}}{\mu_{1}} \int_{-H}^{0} \phi_{1}^{2} \mathrm{~d} z$

This evolution system was rationally derived through a perturbation analysis, and has been amply discussed in our previous work (Sakai and Redekopp, 2010). In that earlier work the model appears with generalizations to include variable topography terms, and inter-modal nonlinear interaction terms, and wind-stress forcing. We purposely excluded wind forcing in Eq. (14) above because, when the forcing is active, higher radial modes (i.e., other than the lowest Poincaré wave mode) are excited and these (noisy) wave modes persist in the domain after the wind ceases. In order to make definitive comparisons with the transport results presented in the previous section, we choose to integrate Eq. (14) forward in time from an initial condition constructed by the sum of the Kelvin and the Poincaré wave modes that correspond to their respective analytical (linear hydrostatic) profiles at the cessation of the wind. Since we consider wind events with moderate strength lasting only some fraction of an inertial period, the effects of nonlinearity and the neglected geostrophic flow during the wind event are expected to be minimal. Therefore, the use of the linear hydrostatic solutions in absence of the geostrophic flow as an initial condition is a reasonable 
choice, and provides a means for direct comparison between the computed transport by an entirely linear dynamics and that associated with a nonlinear hydrodynamic model under similar initial conditions. Furthermore, the approximate representation of the hydrodynamic field at the end of a wind event of limited duration is supported by multiple simulations reported in our earlier work. Adopting this simplification of the initial velocity field, we can restrict our attention to the nonlinear evolution and interaction of the KelvinPoincaré pair within the lowest vertical mode.

The evolution equation set (Eq. 14) with its linear hydrostatic version for the second vertical mode are numerically simulated by employing the spectral method developed in our previous work (Sakai and Redekopp, 2009b), and the tracer equation set (Eq. 13) is simultaneously integrated forward in time with Eq. (14) by employing the fourth-order Runge-Kutta method. The velocity field at the location of each particle is precisely interpolated by exploiting the full range of the spectral expansion functions that are used to approximate the field variables in the spectral method.

Snap shots of cloud evolutions are tabulated for Wedderburn numbers $\mathcal{W}=1.5,1.2$ and 1.0 in Fig. 8. Initial amplitudes of the wave modes are set to the values corresponding to those at the wind cessation time $t_{0}=T_{i} / 3$ for each of the Wedderburn numbers. In all cases the Burger number is fixed to $\mathcal{B}=2$ with the depth-to-radius ratio $\Lambda=1 / 40$, and the stratification profile is set with an epilimnion depth of $h_{2}=1$ and a metalimnion depth of $h_{3}=2$. It is observed from the figure that the cloud boundaries appear to be very similar for the first couple of inertial periods for each Wedderburn number. After three inertial periods, however, a local protrusion forms in the off-shore portion of the cloud (i.e., the region between the outer and the inner radii). This protrusion, extending deeper into the interior of the lake, becomes more intense for smaller Wedderburn numbers (i.e., larger wind stress). Cloud boundaries resulting solely from transport associated with the complete linear hydrostatic field are also included in Fig. 8, and no pronounced off-shore protrusion of the cloud boundary is observed. Clearly, this off-shore burst is induced by nonlinear effects of the background flow field of the first vertical mode.

The off-shore burst is not a phenomenon restricted particularly to the present case with $\mathcal{B}=2$. Similar off-shore transport is observed for different Burger numbers as shown in Fig. $9(\mathcal{B}=1)$ and in Fig. $10(\mathcal{B}=3)$. In these examples the transport associated with the nonlinear dynamics is also compared with that obtained in the presence of the respective linear hydrostatic field. Again, there is no off-shore burst observed in the case of the linear hydrodynamic field. According to the definition of the Burger number (Eq. 7), increasing the Burger number implies an increase in the lake radius (i.e., $\mathcal{B} \propto r_{0}$ ) for a fixed vertical structure (i.e., fixed depth and stratification). For this reason, the Wedderburn numbers for the cases of $\mathcal{B}=1$ and $\mathcal{B}=3$ are adjusted such that the same level of the wind stress $\left(\sim \rho_{0} u_{* 0}^{2}\right)$ is applied to the lake surface with different Burger numbers. The reader should note that both the time $t$ and the inertial period $T_{i}$ are scaled by $r_{0} / N_{0} h_{1}$. Therefore, the snap shots with identical dimensionless time in Figs. 8-10 correspond to identical dimensional time (i.e., $t / T_{i}=t^{*} /\left(r_{0} / N_{0} h_{1}\right) /(2 \pi / B)=$ $t^{*} /\left(r_{0} / N_{0} h_{1}\right) /\left(2 \pi N_{0} h_{1} / f r_{0}\right)=t^{*} /(2 \pi / f)=t^{*} / T_{i}^{*}$, where starred values are dimensional).

For the case of $\mathcal{B}=3$ (Fig. 10) the along-shore transport is slower than that of $\mathcal{B}=2$, and the off-shore burst appears around $t=6 T_{i}$ as compared to $t \approx 4 T_{i}$ for the case of $\mathcal{B}=2$. These differences primarily derive from the fact that the Kelvin wave periods are longer for the larger Burger number, resulting in smaller initial amplitudes of the Kelvin wave at the time of cessation of the wind (i.e., $t_{0}=T_{i} / 3$ in this case). For the case of $\mathcal{B}=1$ (Fig. 8) the along-shore transport does not appear faster than that of $\mathcal{B}=2$, although the off-shore burst appears a bit later $\left(t \approx 6 T_{i}\right)$ than the case of $\mathcal{B}=2$. For $\mathcal{B}=1$ the Kelvin wave of the lowest vertical mode turns out to be superinertial (i.e., $\mathcal{B} / c_{1}<\sqrt{2}$ ). Thus, the residence time of the Kelvin wave is much shorter than that of the subinertial one $(\mathcal{B}=2)$. Also, the radial eigenfuntion of the superinertial Kelvin wave is a regular Bessel function (the same as that of Poincaré waves) as opposed to a modified-Bessel function having an exponential character, with the consequence that the along-shore current is smaller. The smaller currents and shorter residence times of the lowest Kelvin wave mode retard the along-shore transport, as well as the off-shore burst, in lakes with smaller Burger numbers.

It should be added that the region of the along-shore transport for larger Burger number (large lake) is more confined near the basin perimeter relative to the respective lake radius, a direct consequence of the radial width of the Kelvin wave $l_{\mathrm{K}}$ as given by the asymptotic relation $l_{\mathrm{K}} / r_{0} \sim c_{n} / \mathcal{B}$ for large $\mathcal{B}$ (Sakai and Redekopp, 2010). This relation can also explain the fact that the along-shore transport associated with the second vertical mode is more confined near the shore due to the disparately slow eigenspeed $c_{2}$ (this is also evident from Fig. 5).

To visualize the nonlinear effect of the background flow, several snap shots of the isopycnal amplitude $\left(Z_{1}\right)$ of the first vertical mode are exhibited in Fig. 11. Immediately after the wind event $\left(t=0 T_{i}\right)$ the field is linear and appears symmetric, but the Kelvin wave steepens and distorts the symmetry as it propagates in a cyclonic direction. The negative volume of the isopycnal amplitude is gradually concentrated (attracted) to an off-shore side of the Kelvin wave front, and the concentrated negative volume grows into a "solitary-like" wave possessing large amplitude after two inertial periods. We refer here to the solitary-like wave as the "nonlinear Poincaré wave" because this nonlinear wave is particularly concentrated in the off-shore region. The nonlinear Poincaré wave propagates in a cyclonic direction with its phase nearly engaged with the Kelvin wave front (to be precise, the nonlinear Poincaré wave travels slightly faster than the Kelvin 

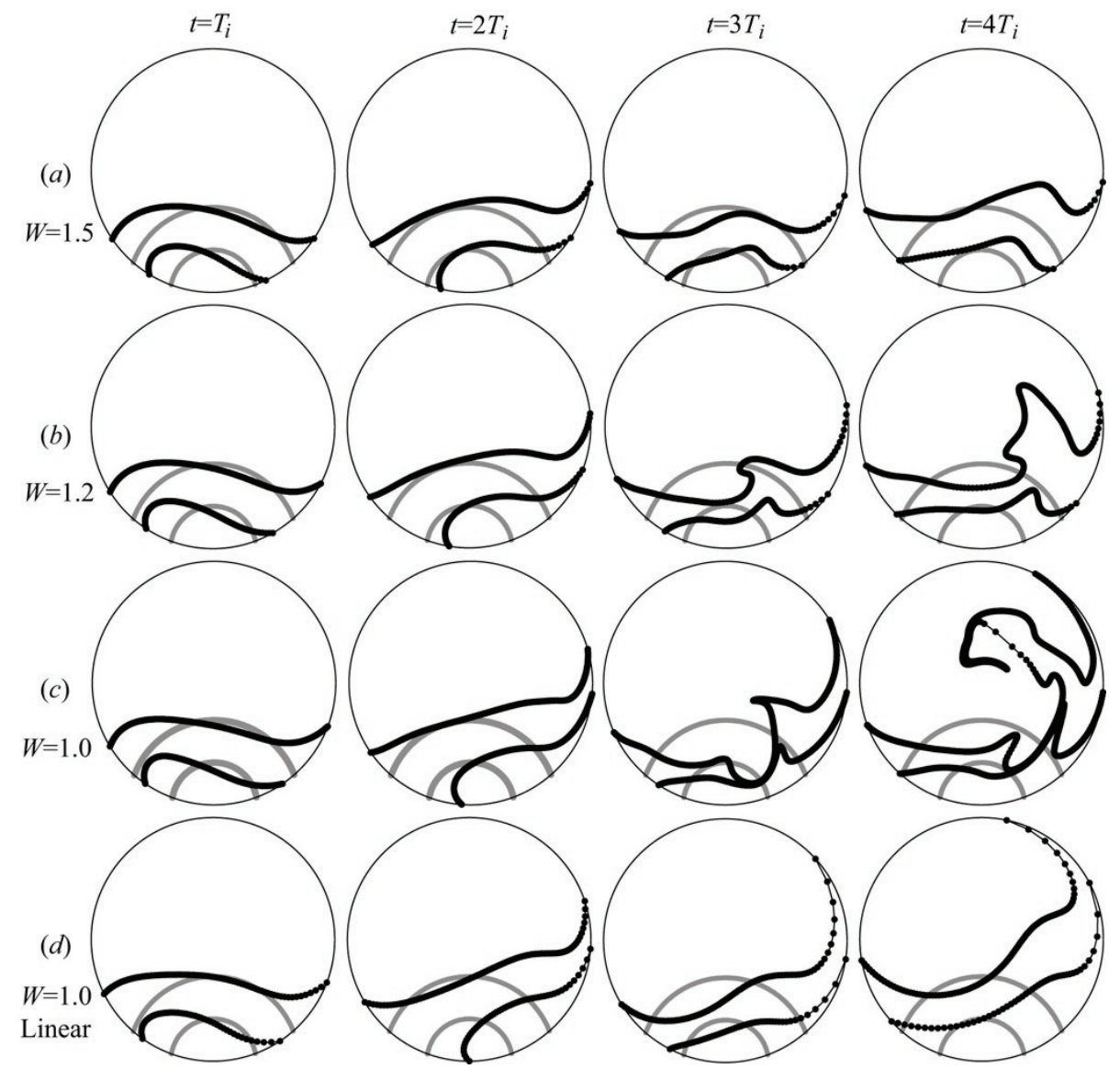

Fig. 8. Evolution of a particle cloud for different Wedderburn numbers $\mathcal{W}=1.5$ (row-a), $\mathcal{W}=1.2$ (row-b) and $\mathcal{W}=1.0$ (rows $\mathbf{c}$, d). Rows (a-c) are subject to nonlinear flow for the lowest vertical mode and linear hydrostatic flow for the second vertical mode. Row (d) is subject to linear hydrostatic flow for both vertical mode-one and two. Grayed points represent the initial position of particles. $\left(\mathcal{B}=2, h_{2}=1, h_{3}=2\right)$.

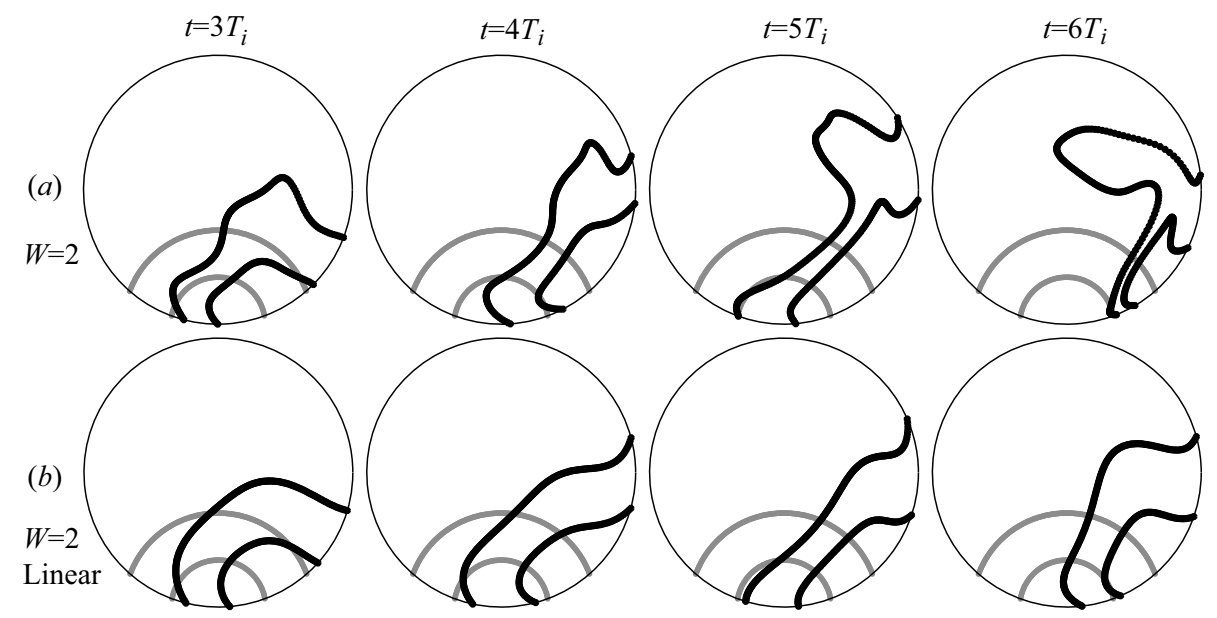

Fig. 9. Evolution of a particle cloud for $\mathcal{B}=1$ with the Wedderburn number $\mathcal{W}=2$. Row (a) is subject to nonlinear flow for the lowest vertical mode and linear hydrostatic flow for the second vertical mode. Row (b) is subject to linear hydrostatic flow for both vertical mode-one and two. Grayed points represent the initial position of particles. $\left(h_{2}=1, h_{3}=2\right)$. 


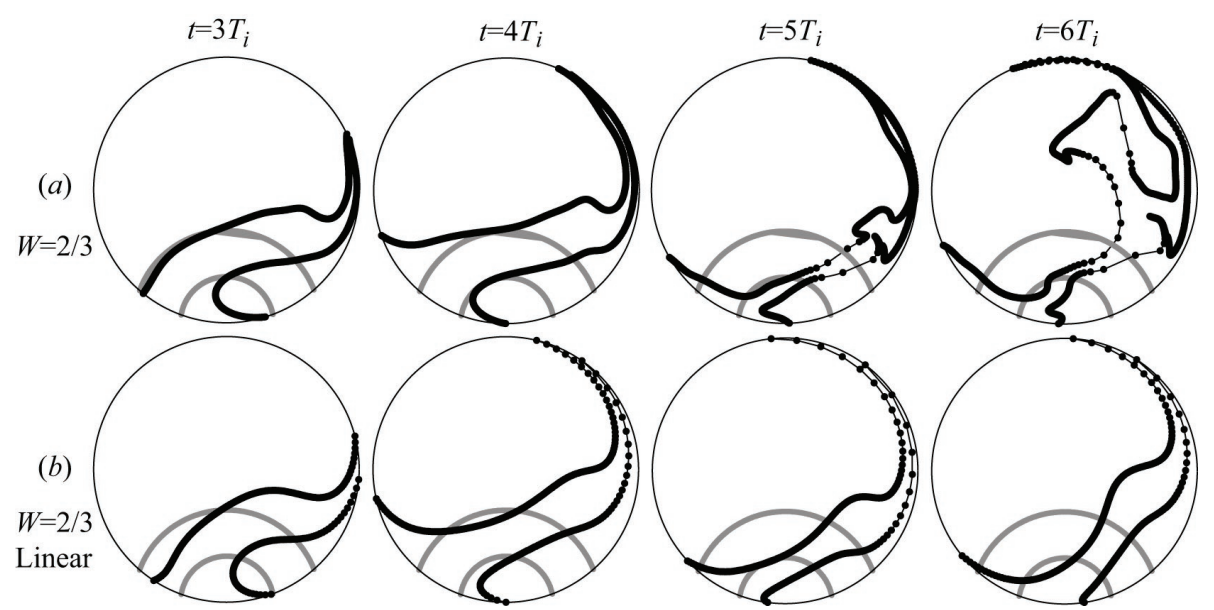

Fig. 10. Evolution of a particle cloud for $\mathcal{B}=3$ with the Wedderburn number $\mathcal{W}=2 / 3$. Row (a) is subject to nonlinear flow for the lowest vertical mode and linear hydrostatic flow for the second vertical mode. Row (b) is subject to linear hydrostatic flow for both vertical mode-one and two. Grayed points represent the initial position of particles. $\left(h_{2}=1, h_{3}=2\right)$

wave because the former wave possesses larger amplitude than the latter one).

In Fig. 12 contour plots of the polar velocity field at the lake top surface are extracted for $t=3 T_{i}$. It can be seen from the figure that large radial and azimuthal velocities are generated by the nonlinear Poincaré wave. This locally concentrated region of large velocities travels around the basin, and it passes across the off-shore portion of the cloud patch, eventually causing the inner portion of the cloud to burst in the off-shore direction.

An off-shore current can be generated in a region of a steepened Kelvin wave front as given in the earlier works of Fedorov and Melville (1995, 1996 for the Kelvin jump), where the hydrostatic, nonlinear evolution models describing the Kelvin wave (or jump) propagation along a rectilinear coastline in a semi-infinite domain are employed. In the present model we find that a similar off-shore current is generated in a slightly off-shore region of the steepened Kelvin wave front. However, such an off-shore current is smaller than the along-shore counterpart by an order of magnitude. The off-shore current of the nonlinear Poincaré waves can exceed the along-shore current of the steepened Kelvin wave. In the present model, therefore, the contribution of the Kelvin wave-generated off-shore current to the off-shore transport is far less relative to that derived from the nonlinear Poincaré waves.

The results presented in this section should be viewed with some caution because the amplitudes of the fully-evolved Poincaré waves grow quite large (e.g., in Fig. 11 the amplitude grows to about twice as large as the epilimnion depth $h_{1}$ ). With such large amplitude the magnitude of the velocity field is potentially an overestimate with use of the weakly-nonlinear model presented here. It is widely known that the higher order nonlinearity has the effect of limiting the amplitudes of solitary waves (e.g., see Helfrich and Mellville, 2006; Sakai and Redekopp, 2007). Nevertheless, the initial stage of the generation process of the nonlinear Poincaré wave presented here is still valid because the wave amplitude at that stage remains modest.

If the field consists solely of either a Kelvin or a Poincaré wave, the nonlinear Poincaré wave does not emerge (see Figs. 5 and 15 in Sakai and Redekopp, 2010). It is known that the amplitude of the Poincaré wave undergoes an oscillatory, temporal modulation in nonlinear flows (Sakai and Redekopp, 2010; de la Fuente et al., 2008). We conjecture that the nonlinear modulation of the Poincaré wave is amplified through the nonlinear interaction with the steepened Kelvin wave, resulting in the nonlinear Poincaré wave of large amplitude as presented in this section.

\section{Conclusions}

In this paper we considered the effects of the second vertical mode and the effects of nonlinearity of the energetically dominant flows on the transport of Lagrangian particles in an ideal circular cylindrical lake subject to a uniform wind stress persisting for a finite duration.

It is confirmed that the Kelvin waves primarily drive the along-shore transport of particles near the basin perimeter, and that the Poincaré waves are the primary source of driving the off-shore transport. It is quite common to neglect the dynamics of the second or higher vertical eigenmodes in some flow models because of their perceived insignificant amplitudes relative to the amplitude of the energetically dominant eigenmode (usually the lowest vertical mode). However, when it comes to study the particle transport, one should not ignore the second vertical mode in the background flow domain because the disparately slow Kelvin wave of the second 

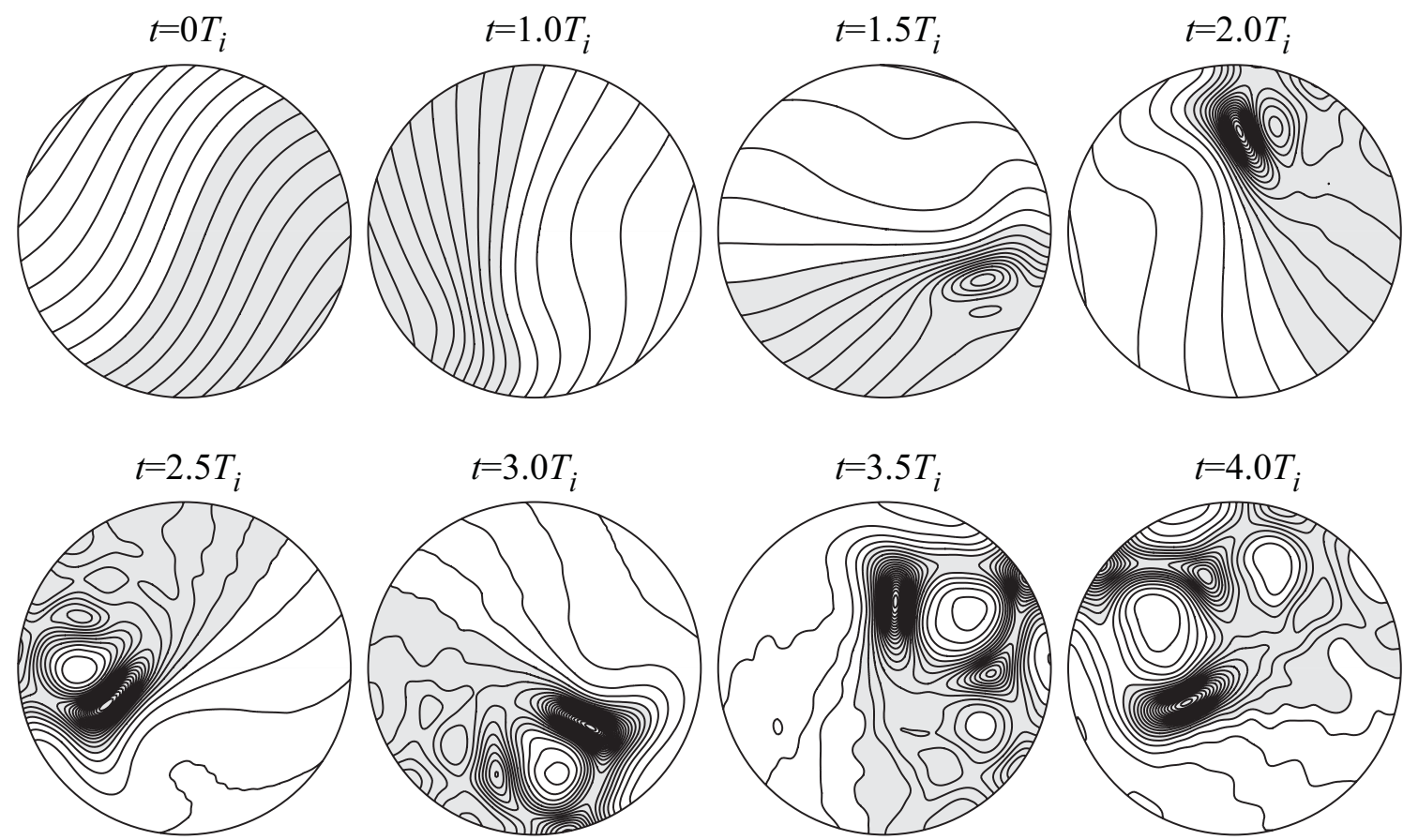

Fig. 11. Nonlinear evolution of the isopycinal amplitude $Z$ of the first vertical mode. Contour level step is every 0.1 dimensionless unit from zero values, and shaded regions represent negative values. $\left(\mathcal{B}=2, \mathcal{W}=1.2, h_{2}=1, h_{3}=2\right)$.

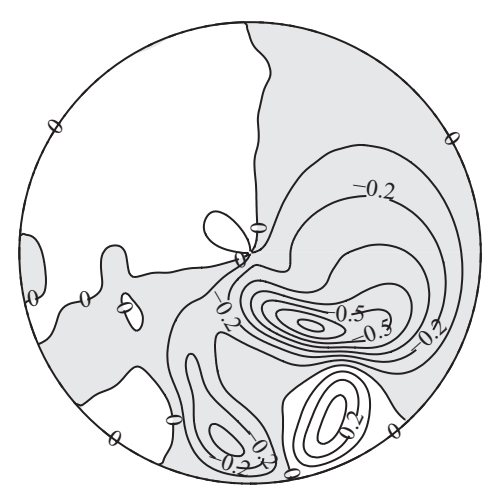

Radial velocity

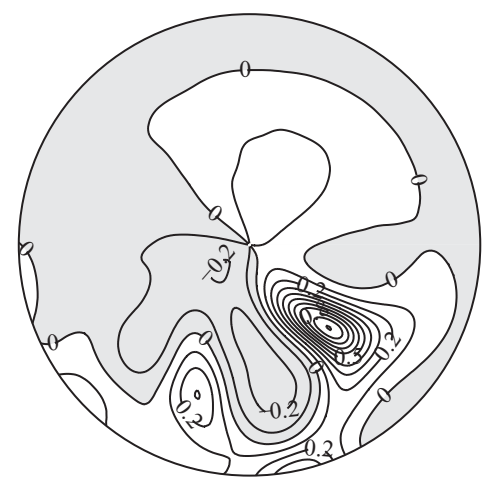

Azimuthal velocity

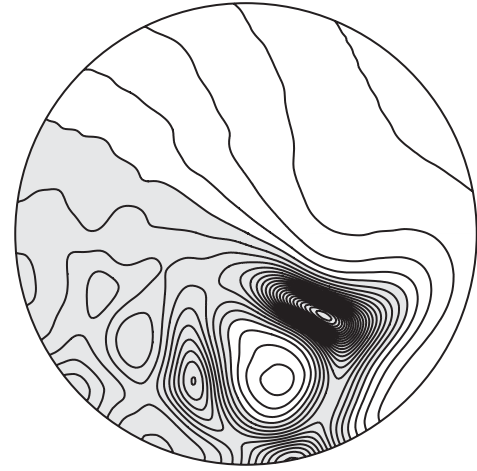

V1 Isopycnal amplitude

Fig. 12. : Instantaneous velocity components at the upper surface of the lake for $\mathcal{B}=2, \mathcal{W}=1.2$ at $t=3 T_{i}$ along with the corresponding isopycnal amplitude of the first vertical mode taken from Fig. 11. Contour level step is every 0.1 dimensionless unit, and shaded regions represent negative values. $\left(h_{2}=1, h_{3}=2\right)$.

vertical mode is resident for a long time and, then, the resultant particle transport becomes non-negligible and comparable to the transport driven by the first vertical mode.

Effects of the flow nonlinearity become increasingly important as time increases. When both the Kelvin and the Poincaré waves coexist in the lake domain, a solitary-like wave of large amplitude (i.e., the nonlinear Poincaré wave) can emerge in an off-shore region as a consequence of nonlinear interaction between the Kelvin-Poincaré wave pair. The nonlinear Poincaré wave co-propagates with the Kelvin wave in a cyclonic direction nearly side by side, and the large current generated by the nonlinear Poincaré wave potentially induces a pronounced off-shore transport of a particle cloud and, therewith, enhances horizontal mixing. This quite interesting phenomenon is an entirely nonlinear effect and cannot be derived from the classical linear hydrostatic model.

Since we employed a weakly-nonlinear model to drive the background flow, the largeness of the amplitude of the nonlinear Poincaré wave remains in question. Similar flow nonlinearity is also possible in the flow domain of the second 
vertical mode. Nonlinear interaction between the lowest two vertical modes is certainly possible (e.g., Sakai and Redekopp, 2009a). We restricted our study to the particle transport on the top (or bottom) surface of the simplified lake model. While particle transport is three-dimensional in nature, and since it is almost certainly significantly affected by three-dimensional bathymetric irregularities (e.g., Okely et al., 2010; implications given by Wake et al., 2005), vertical motions and the effect of irregular lake boundaries on particle transport are important issues deserving of future study in order to augment the conclusions of this present work.

Edited by: R. Grimshaw

Reviewed by: two anonymous referees

\section{References}

Csanady, G. T.: Motions in a model Great Lake due to a suddenly imposed wind, J. Geophys. Res., 73, 6435-6447, 1968.

Csanady, G. T.: Response of large stratified lakes to wind, J. Phys. Oceanogr., 2, 3-13, 1972.

Csanady, G. T.: Hydrodynamics of large lakes, Ann. Rev. Fluid Mech., 7, 357-386, 1975.

de la Fuente, A., Shimizu, K., Imberger, J., and Niño, Y.: The evolution of internal waves in a rotating, stratified, circular basin and the influence of weakly nonlinear and nonhydrostatic accelarations, Limnol. Oceanogr., 53, 2738-2748, 2008.

Fedorov, A. V. and Mellville, W. K.: Hydrolic jumps at boundaries in rotating fluids, J. Fluid Mech., 324, 55-82, 1996.

Fedorov, A. V. and Melville, W. K.: Propagation and breaking of nonlinear Kelvin waves, J. Phys. Oceanogr., 25, 2518-2531, 1995.

Helfrich, K. R. and Mellville, W. K.: Long nonlinear internal waves, Annu. Rev. Fluid Mech., 38, 395-425, doi:10.1146/annurev.fluid.38.050304.092129, 2006.
Horn, D. A., Imberger, J., and Ivey, G. N.: The degeneration of large-scale interfacial gravity waves in lakes, J. Fluid Mech., 434, 181-207, 2001.

Imberger, J.: Flux paths in stratified lake: A review, vol. 54 of "Physical processes in lakes and oceans: coastal and esturine studies", American Geophysical Union, Washington, DC, 1-17, 1998.

Monismith, S.: Modal response of reservoirs to wind stress, J. Hydraul. Eng., 113, 1290-1306, 1987.

Okely, P., Imberger, J., and Shimizu, K.: Particle disparsal due to interplay motions in the surface layer of a small reservoir, Limnol. Oceanogr., 55, 589-603, 2010.

Patil, S., Singh, V. P., and Imberger, J.: Horizontal dispersion in gyres-interal wave flow field in a rotating circluar lake, J. Hydrol. Eng., 15, 597-611, 2010.

Sakai, T. and Redekopp, L. G.: Models for strongly-nonlinear evolution of long internal waves in a two-layer stratification, Nonlin. Processes Geophys., 14, 31-47, doi:10.5194/npg-14-31-2007, 2007.

Sakai, T. and Redekopp, L. G.: A weakly nonlinear model for multi-modal evolution of wind-generated long internal waves in a closed basin, Nonlin. Processes Geophys., 16, 487-502, doi:10.5194/npg-16-487-2009, 2009 a.

Sakai, T. and Redekopp, L. G.: An application of onesided Jacobi polynomial for spectral modeling of vector field in polar coordinates, J. Comput. Phys., 228, 7069-7085, doi:10.1016/j.jcp.2009.06.017, 2009b.

Sakai, T. and Redekopp, L. G.: A weakly nonlinear evolution model for long internal waves in a large lake, J. Fluid Mech., 656, 260297, doi:10.1017/S0022112010001114, 2010.

Stocker, R. and Imberger, J.: Energy partitioning and horizontal dispersion in a stratified rotating lake, J. Phys. Oceanogr., 33, 512-529, 2003.

Wake, G. W., Ivey, G. N., and Imberger, J.: The temporal evolution of baroclinic basin-scale waves in a rotating circular basin, J. Fluid Mech., 523, 367-392, doi:10.1017/s0022112004002344, 2005. 